\title{
Software-Defined Networking for Ubiquitous Healthcare Service Delivery
}

\author{
Foteini Andriopoulou ${ }^{1}$, Konstantinos Birkos ${ }^{1}$, Georgios Mantas ${ }^{2,3}$ and Dimitrios Lymber- \\ opoulos 1 \\ ${ }^{1}$ Department of Electrical and Computer Engineering, University of Patras, Patras, 26500 Greece \\ ${ }^{2}$ Faculty of Engineering and Science, University of Greenwich, UK \\ ${ }^{3}$ Instituto de Telecomunicações - Aveiro, Portugal \\ fandriopdupatras.gr, kmpirkosdece.upatras.gr, \\ dlymbero@upatras.gr \\ G.Mantas@greenwich.ac.uk
}

\begin{abstract}
The growth of the mobile, portable devices and the server-to-server communication through cloud computing increase the network traffic. The dependence of the ubiquitous healthcare service delivery on the network connectivity creates failures that may interrupt or delay the treatment plan with adverse effects in patients' quality of life even leading to mortality. In the present work, we propose the incorporation of Software Defined Networking (SDN) features in the healthcare domain in order to provide the appropriate bandwidth and guarantee the accurate real time medical data transmission independently of the connectivity of the ISP provider. The SDN controller monitors the network traffic and specifies how traffic should be routed providing load balancing, lower delays and better performance. Finally, the proposed healthcare architecture addresses the SDN scalability challenge by incorporating the logically centralized control plane using multiple distributed controllers. A 2-tier hierarchical overlay is formed among SDN controllers following the principles of peer-to-peer networking.
\end{abstract}

Keywords: Software Defined Network, Body Area Networks, Ubiquitous Healthcare Service Delivery

\section{Introduction}

The explosion of internet and mobile applications with the evolution of the pervasive environment, during the last few years, have dramatically changed the way that healthcare is delivered. Wireless and mobile technologies have enabled real time data transmission through numerous portable devices, sensors and other computing entities in order to access care remotely and receive fast treatment in emergency cases. This explosive growth of data transmission introduces many challenges in terms of data storage, interoperability and availability of resources. Cloud computing approach was adopted to address the aforementioned issues [5].

However, the rapid growth of storage resources in the cloud platforms and the need for efficient access to these resources has increased the requirements for fast underlying networks. While healthcare services become more dependent on the network connectivity, network failures may interrupt or delay the healthcare service delivery with adverse effects in patients' quality of life even leading to mortality. In this context, more than ever is 
required a flexible, scalable and agile network that simplifies the network configuration and management. Software Defined Networking (SDN) [17] is the most promising solution for increasing network utilization and reducing hardware complexity and costs. SDN is a networking architecture that provides network intelligence out of the switching devices by abstracting the logical part of the computer networks and placing it on the controllers [17], [16], [9].

In large scale networks such as ubiquitous healthcare network, the controller could become a bottleneck with negative effects on network maintenance and data forwarding. Multiple solutions have been proposed for avoiding the increased traffic in the controller. Some of them propose the insertion of multiple controllers into the network architecture for replacing the disconnected controllers, while some other propose two levels of hierarchy in order to share responsibilities among cotrollers [18]. DevoFlow [2] seperates the flows in two classes, the short-lived flows and the long-lived for reducing the tasks executed by the controller. Only the long lived flows were forwarded to the controller where the short lived flows are handled in the data plane. HotSwap [14] proposed a mechanism through which a controller is able to be replaced by another new controller on-the fly without any disruption of the network. However, the error possibilities are many as well as this process introduces further delays. Koponen et al. [6] proposed Onix platform, a distributed control platform on top of which a network control plane can be implemented. It provides flexible distribution components that enable software designers to implement control applications without re-inventing distribution mechanisms [6]. Tootoonchain et Ganjali proposed HyperFlow [13], a logically centralized but physically distributed event-based control plane. It allows deployment of any number of controllers which share the same broad network view. They serve requests without active contact of any remote node that decrease the delays and the flow installation time. Kandoo [4], provides a distributed control plane with two levels of hierarchy for the controllers, the local controllers and the root controllers. The local controllers are located closer to the data path in order to receive requests from the switches while the root controllers control the local switches and handles applications that require network wide knowledge.

In the present work, we propose the incorporation of the SDN in both the access and core network of the ubiquitous healthcare system architecture in order to achieve network intelligence. Incorporating the SDN in the access network, the network devices of the end users (patients) provide access to the core network where the SDN controller classifies the packets. These packets are prioritized over the network. Then, the SDN controller which has broader knowledge of the network status is able to take decisions for routing or redirecting the vital bio-signals and other essential medical data over neighbor paths providing the appropriate bandwidth for the accurate real time data transmission. The proposed healthcare network architecture incorporates a logically centralized control plane using multiple distributed controllers. These controllers constitute a large-scale distributed system following the principles of peer-to-peer networking [11]. The maintenance of the distributed multiple controllers based on the peer-to-peer paradigm provides scalability, robustness and low response time. A 2-tier hierarchical overlay is proposed. It enables efficient cooperation and ensures the dynamic communication and information exchange through the ISP and healthcare providers.

The rest of the paper is organized as follows: Section 3 presents the basic architecture of the healthcare network enhanced with the SDN principles. It provides the basic use 
cases for the mobile and wireless networks or access the network and how the MPLS functionality is implementing for these purposes. Finally, Section 3 concludes the present work.

\section{Healthcare Network Architecture over SDN}

\subsection{Overview}

Wireless sensor networks and BANs are considered fundamental for supporting ambient living and providing high-quality healthcare service delivery [10], [7]. Fig. 1 presents an overview of the healthcare environment with the involved entities.

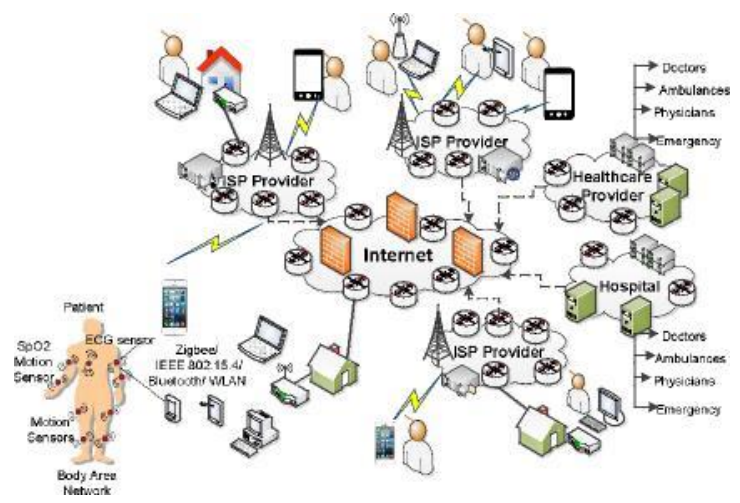

Fig. 1. An overview of the ubiquitous healthcare environment.

The patient uses wearable and mobile sensors that acquire bio-signal information and transmit it to a control device. The control device aggregates all the signals from the sensors and forwards them via an ISP [15] for further processing to healthcare providers. Healthcare providers provide services for ubiquitous patient monitoring and have their own network that can be a legacy network or based on SDN/OpenFLow principles. Fig. 2 presents the system overview of the ubiquitous healthcare network based on SDN. 


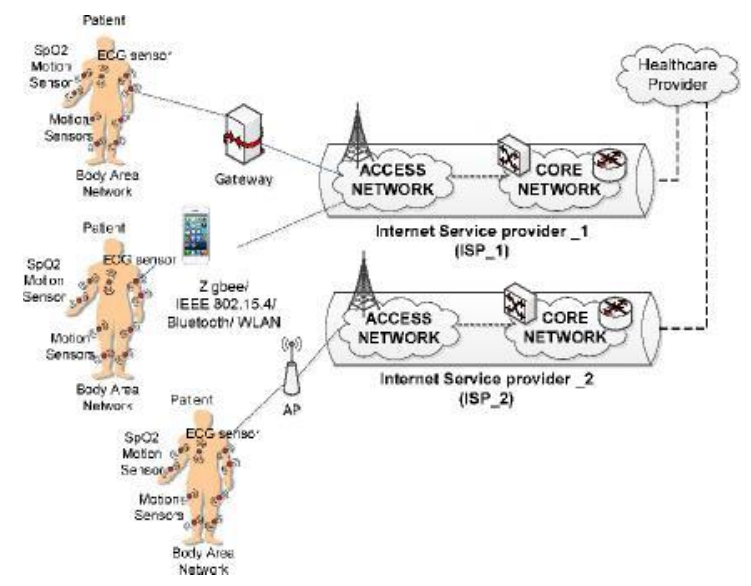

Fig. 2. The overview of the healthcare network.

The multiple controllers of the ISPs and healthcare providers are organized into a 2-tier hierarchical overlay network following the peer-to-peer paradigm [11]. Each ISP and healthcare provider organizes its controllers peers in an overlay. Peers in the same overlay establish and maintain logical connections with other peers, perform overlay routing, store and retrieve information such as available resources, network traffic, etc. Peers with enhanced computational capabilities and network resources become super-peers. Superpeers from different overlays are further organized into an overlay and are responsible for handling requests among peers that belong to different overlays. In order to avoid single points of failure, there are multiple super-peers in each overlay. Bootstrap peers are responsible for overlay formation and maintenance. In this hierarchical scheme, requests are propagated in a smaller number of ISPs and controllers, achieving low response time. Fig. 3 presents the formation of the 2-tier hierarchical overlay network.

\subsection{Basic Architecture}

Each healthcare provider is responsible to check periodically the reception of vital biosignals from the subscribed patients. If no bio-signals have been received within a predefined threshold duration or if the received data rate is below an expected value, the healthcare provider contacts the Bootstrap peer and sends a request with information related to the required QoS level (minimum bitrate, delay, packet loss) for the acceptable provisioning of the healthcare services. Bootstrap peer forwards the request to the ISP providers in order in order to find to which ISP the patient is subscribed. The ISP providers forward further the request to their overlay and a success or failure response is sent to the Bootstrap. The ISP is informed about the healthcare requirements and initiates a set of actions towards the restoration of network access. 


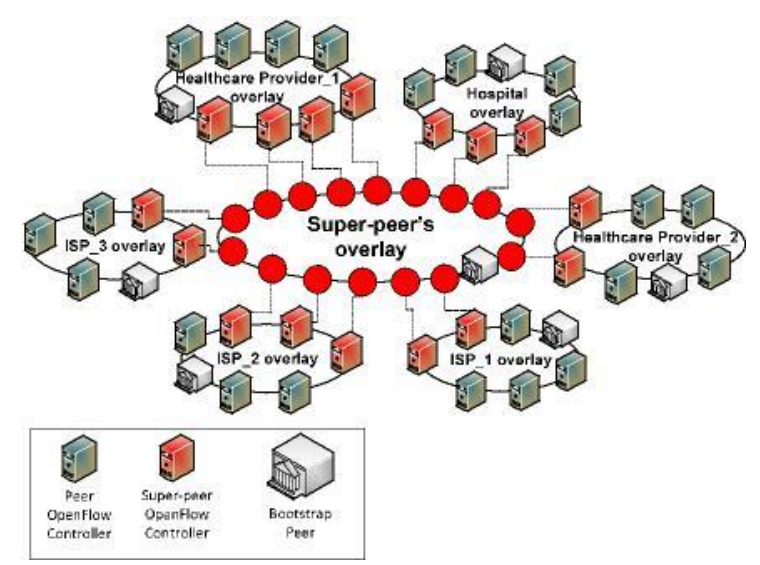

Fig. 3. The 2-tier hierarchical overlay formation

However, a patient may not have an Internet connection or his connectivity may be intermittent due to mobility. If limited or no connectivity is observed, the ISP executes a set of actions to recover its network connectivity or may request to cooperate with another ISP in order to support the patient. In the following section, we analyze two possible ways that an ISP may execute for network access restoration based on the SDN functionality: the wireless network restoration through WiFi access points and the enhancement of LTE mobile access.

Wireless Network Access In this section, we propose how the ISP can provide enhanced wireless network access to the patients. In this context, patients carry WiFi-enabled devices while their ISP operates a set of OpenFlow-compatible WiFi APs controlled by one or multiple controllers. The controller interacts with specialized applications for network management. The healthcare provider may also have a set of public APs which can be used to assist patients for medical data transmission. A special interface provides communication with the ISPs of the registered users.

The network management application that runs on top of the controller of the ISP tries to find the patient's location based on his recent activity, global positioning information from his device or geo-location information from the cellular network. Then it selects a set of candidate APs that can be used for connecting the user to the network. The controller of the ISP has global knowledge of the network connections and their status. Therefore, it can assess the status of the connection between each AP and the healthcare provider. Based on this information, the AP that meets the constraints better is selected. The controller installs a rule inside this AP that permits connectivity to the MAC address of the device of the patient. At this point, the device can access the network and transmit data to the healthcare provider. Fig. 4 depicts the proposed setup. 


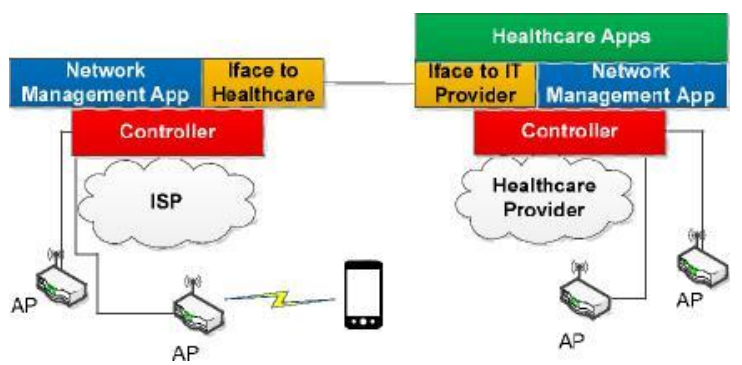

Fig. 4. SDN-based wireless network access for healthcare data flows.

However, public APs or APs connected to other ISPs may also be available within the area of the patient. In order to support cooperation for medical data transmission, the healthcare providers and the ISPs are interconnecting through the aforementioned super-peer's overlay as presented in Fig. 5. In this cooperative scheme, if the patient's ISP cannot provide any active APs appropriate for the bio-signal transmission, it informs the Bootstrap peer which broadcasts a request through the super-peer's overlay to other ISPs for requesting access. Each ISP's super-peer forward the request to its internal overlay in order to search for available network resources. The controllers (peers) of each ISP overlay check the available APs and report back to the ISP's super-peer. The ISPs' super-peers response to the Bootstrap with a message that contains the active and available APs. Bootstrap peer compares the candidate APs, selects the one that meets the QoS constraints better and sends a request to the corresponding ISP. Then the controller instructs the AP to accept connection of the patient's device and permit traffic flows towards the IP of the healthcare provider.

Mobile Network Access. In this section, the proposed solution for enhanced mobile network access for healthcare purposes is presented. In this case, bio-signals are sent to the healthcare provider via an OpenFlow-enabled LTE cellular network[12], [1], [8].

In the proposed system, the Serving Gateways (S-GWs) consist of two distinct parts: a data-plane part and a control-plane part. The control plane is actually removed from $S$ GW and it is implemented by the controller of the Mobile Network Operator (MNO). In the same manner, the control plane of the Packet Data Network (PDN) Gateway (P-GW) may also be realized by means of a controller. In this case, the necessary interaction between S-GW and P-GW occurs through the communication of the corresponding controllers. On top of the controllers, specialized applications perform network monitoring, control, radio resource management and mobility management. Therefore, the Mobility Management Entity (MME) is also part of the software on top of the controller. Fig. 6 shows how mobile network access for healthcare data flows is provided through an SDNenabled LTE network. 


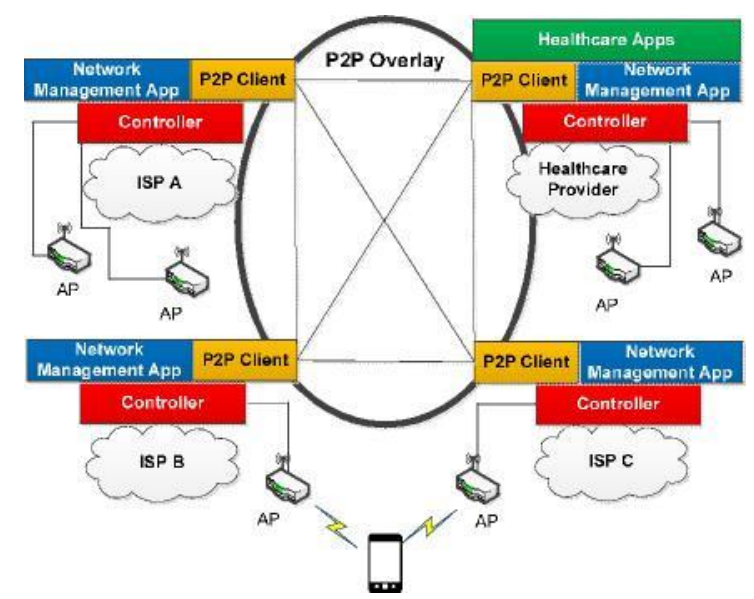

Fig. 5. . Peer-to-peer overlay for wireless network access across multiple ISPs.

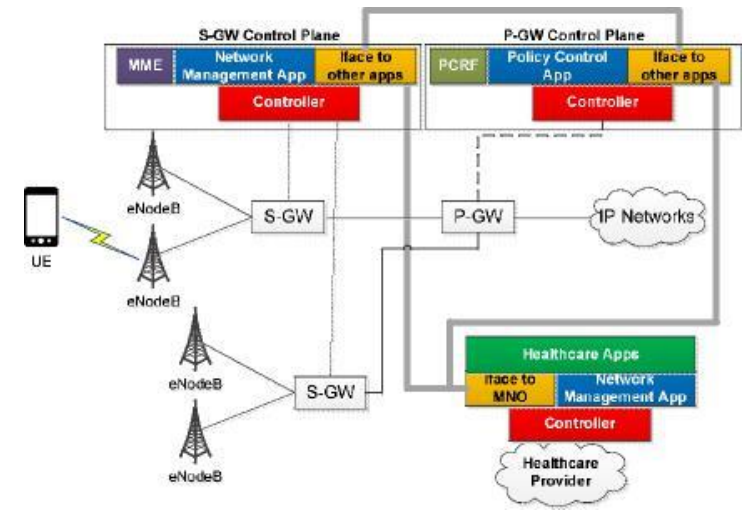

Fig. 6. SDN-based mobile network access for healthcare data flows.

In order to provide specialized access policies to healthcare users, the MNO must be aware of the set of these users and the related QoS requirements. This information is provided by the healthcare provider via the super-peer's overlay (P2P overlay) described in the previous section. The network management application executed on top of the controller of an S-GW has complete knowledge of the devices producing healthcare data flows that are connected to the eNodeBs of its jurisdiction. This global visibility enables MNOs to favor healthcare applications more easily and more efficiently.

If the users experience poor signal strength, the serving eNodeBs are dictated by the controller to selectively increase the power allocated to the corresponding sub-channels. In addition, the network management application can protect devices running healthcare applications from interference and treat them differently. When performing Inter-Cell Interference Coordination (ICIC), the sub-channels allocated to these specific devices can be excluded from power reduction. Under increased user density, enhanced ICIC (eICIC) can create pico-cells in certain overloaded areas to cover the devices that communicate with the healthcare provider.

It is also possible that in certain rural areas the user has too poor coverage from its own operator. In this case, it can be served by alternative MNOs that may provide better 
coverage. This scenario requires cooperation among multiple operators and/or the healthcare provider. Cooperation and coordination is realized by means of the peer-topeer overlay of providers. When poor or no coverage at all is observed, the current MNO broadcasts a request to the peer-to-peer overlay. After a negotiation among candidate MNOs, the best MNO in terms of coverage and traffic load is selected to serve the user. Fig. 7 depicts the peer-to-peer overlay for mobile network access across multiple MNOs.

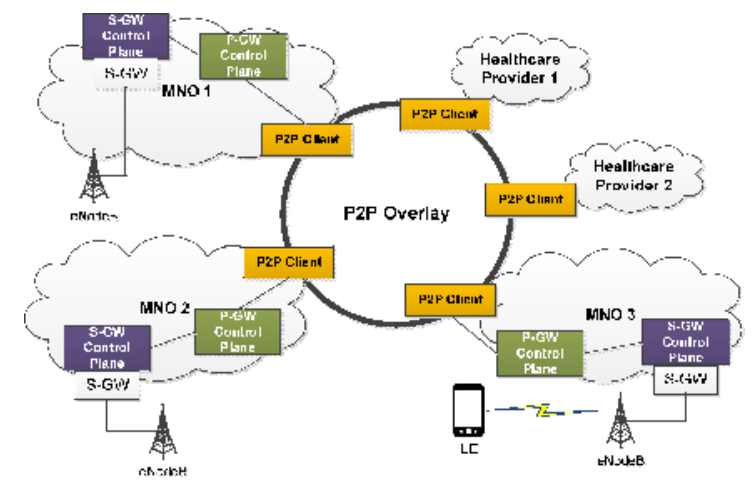

Fig. 7. Peer-to-peer overlay for mobile network access across multiple MNOs.

SDN-based MPLS Functionality. Inside the core network of each provider (ISP, MNO or healthcare provider), MPLS is used for traffic engineering purposes in order to provide the appropriate bandwidth, QoS and access control to the healthcare data flows. However, in our model, MPLS is controlled by software modules, according to the SDN paradigm. The introduction of SDN control in MPLS networks has significant advantages. A major advantage is that SDN-based MPLS provides better control over the formation and maintenance of Label-Switched Paths (LSPs). In addition, network dynamics induce churn effects which are difficult to be handled by conventional MPLS which lacks global knowledge and centralized control [3].

In the proposed system, the MPLS control plane resides on top of centralized controllers. It has a holistic view of the active flows of healthcare data which may enter the network via different ingress routers. Each time a healthcare data flow is initialized, the MPLS control plane selects or forms an MPLS tunnel that meets certain bandwidth, delay and packet loss constraints. The global network view offered by the controller enables the MPLS control plane to create routes that meet multiple criteria efficiently. Any change in the network topology or the status of the links is resolved by the MPLS control plane with minimal messaging overhead.

Due to auto-route and auto-bandwidth features of MPLS, flows may need to migrate to different LSPs or existing LSPs may need to be reconstructed. The MPLS control plane protects the healthcare data flows from frequent changes and prioritizes them in the bandwidth reservation inside the MPLS tunnels. Moreover, the network access of multiple concurrent healthcare data flows is globally optimized. QoS requirements of individual flows as well as fairness among competing flows are the objectives of the optimization process. 


\section{Conclusion}

In the present work, we proposed the use of the SDN in both the access and core network of the ubiquitous healthcare system architecture in order to achieve network intelligence in favor of healthcare service provisioning. In the proposed architecture, multiple distributed controllers constitute a large-scale hierarchical peer-to-peer overlay which enables efficient cooperation and information exchange among ISPs and healthcare providers. We have shown how this interaction can lead in proper transmission of the medical data by enhancing admission control, prioritization, network resource management and traffic shaping.

It is our strong belief that the proposed ubiquitous healthcare network enhanced with SDN features and strengthened with the peer-to-peer paradigm is able to: a) help the endusers to overcome some network restrictions in order to transfer their vital measurements and b) support the communication and collaboration of the ISP and healthcare providers in an efficient and scalable manner. As a future work the proposed network platform will be emulated using Mininet and open source controllers in order to study the opportunistic behavior of the system in real time conditions.

\section{References}

1. Brief, O.S.: Openflow-enabled mobile and wireless networks (2013)

2. Curtis, A.R., Mogul, J.C., Tourrilhes, J., Yalagandula, P., Sharma, P., Banerjee, S.: Devoflow: scaling flow management for high-performance networks. In: ACM SIGCOMM Computer Communication Review. vol. 41, pp. 254-265. ACM (2011) 
3. Das, S., Sharafat, A., Parulkar, G., McKeown, N.: Mpls with a simple open control plane. In: Optical Fiber Communication Conference. p. OWP2. Optical Society of America (2011)

4. Hassas Yeganeh, S., Ganjali, Y.: Kandoo: a framework for efficient and scalable offloading of control applications. In: Proceedings of the first workshop on Hot topics in software defined networks. pp. 19-24. ACM (2012)

5. Hogan, M., Liu, F., Sokol, A., Tong, J.: Nist cloud computing standards roadmap. NIST Special Publication 35 (2011)

6. Koponen, T., Casado, M., Gude, N., Stribling, J., Poutievski, L., Zhu, M., Ra-manathan, R., Iwata, Y., Inoue, H., Hama, T., et al.: Onix: A distributed control platform for large-scale production networks. In: OSDI. vol. 10, pp. 1-6 (2010)

7. Lee, Y.D., Chung, W.Y.: Wireless sensor network based wearable smart shirt for ubiquitous health and activity monitoring. Sensors and Actuators B: Chemical 140(2), 390-395 (2009)

8. Li, L.E., Mao, Z.M., Rexford, J.: Toward software-defined cellular networks. In: Software Defined Networking (EWSDN), 2012 European Workshop on. pp. 7-12. IEEE (2012)

9. McKeown, N., Anderson, T., Balakrishnan, H., Parulkar, G., Peterson, L., Rexford, J., Shenker, S., Turner, J.: Openflow: enabling innovation in campus networks. ACM SIGCOMM Computer Communication Review 38(2), 69-74 (2008)

10. Pandian, P., Mohanavelu, K., Safeer, K., Kotresh, T., Shakunthala, D., Gopal, P., Padaki, V.: Smart vest: Wearable multi-parameter remote physiological monitoring system. Medical engineering \& physics 30(4), 466-477 (2008)

11. Risson, J., Moors, T.: Survey of research towards robust peer-to-peer networks: Search methods. Computer networks 50(17), 3485-3521 (2006)

12. Said, S.B.H., Sama, M.R., Guillouard, K., Suciu, L., Simon, G., Lagrange, X., Bonnin, J.M.: New control plane in 3gpp lte/epc architecture for on-demand connectivity service. In: Cloud Networking (CloudNet), 2013 IEEE 2nd International Conference on. pp. 205-209. IEEE (2013)

13. Tootoonchian, A., Ganjali, Y.: Hyperflow: A distributed control plane for openflow. In: Proceedings of the 2010 internet network management conference on Research on enterprise networking. pp. 3-3. USENIX Association (2010)

14. Vanbever, L., Reich, J., Benson, T., Foster, N., Rexford, J.: Hotswap: correct and efficient controller upgrades for software-defined networks. In: Proceedings of the second ACM SIGCOMM workshop on Hot topics in software defined networking. pp. 133-138. ACM (2013)

15. Vouyioukas, D., Maglogiannis, I.: Communication issues in pervasive healthcare systems and applications. Pervasive and Smart Technologies for Healthcare: Ubiquitous Methodologies and Tools pp. 197-227 (2010)

16. Wang, W., Haas, R., Salim, J.H., Doria, A., Khosravi, H.M.: Forwarding and control element separation (forces) protocol specification (2010)

17. Xia, W., Wen, Y., Foh, C.H., Niyato, D., Xie, H.: A survey on software-defined networking (2014)

18. Yeganeh, S.H., Tootoonchian, A., Ganjali, Y.: On scalability of software-defined networking. Communications Magazine, IEEE 51(2), 136-141 (2013) 